\title{
The Changes of Serum Lipoprotein(a) and Plasminogen Activator Inhibitor-1 in Primary Nephrotic Syndrome
}

\section{Zhan-zhu Lv1 ${ }^{1}$, Chun-xi Zheng², Rong-rong Dou ${ }^{3}$, Bin Han ${ }^{3}$, Run-hua Tian $^{3}$, Yan Wei ${ }^{4}$}

\author{
${ }^{1}$ Department of Biochemistry and Molecular Biology, Medical College of Qingdao University; \\ ${ }^{2}$ The commercial stuff hospital of Qingdao; \\ ${ }^{3}$ The affiliated hospital of Qingdao University; \\ ${ }^{4}$ Department of Histology and Embryology, Medical College of Qingdao University, Qingdao 266021, Shandong, \\ China
}

*Supported by a grant from Shandong Science and Technology Development Plan (2012-YD-18037)

\begin{abstract}
Objective To investigate the changes of serum lipoprotein(a) [Lp(a)] and plasminogen activator inhibitor-1 (PAI-1) in primary nephrotic syndrome (PNS). Methods 60 PNS cases whose renal pathological types were identified and 57 healthy physical examination people as normal controls (NC) were selected. Automatic biochemical analyzer detected the levels of serum Lp(a) and blood lipids. Enzyme-linked immunosorbent (Elisa) method tested the concentration of serum PAI-1. The nonparametric Spearman rank coefficient test was used to analyze correlations between variables. Results Compared with the NC group, Lp(a) and PAI-1 were significantly higher in PNS group $(P<0.05)$. Lp(a), PAI-1 and lipids were found negatively related with albumin in PNS group. Lp(a) was positively correlated with PAI-1 in PNS patients, and the Spearman rank coefficient was $0.381(P=0.003)$ Binary logistic regression analysis results showed that $\mathrm{Lp}(\mathrm{a})$ and PAI-1 were two risk factors in group PNS. Conclusions Lp(a) and PAI-1 concentrations are increased obviously in PNS patients, and Lp(a) was positively correlated with PAI-1 in PNS patients. They could be two risk factors of PNS patients.
\end{abstract}

Keywords: Lipoprotein(a); Plasminogen Activator Inhibitor-1; Primary Nephrotic Syndrome

\section{Introduction}

Clinical diagnosis and treatments found that the damages of kidney endothelial cells, micro vascular thrombosis and blood coagulation fibrinolytic system changes are closely associated with the occurrence and development of kidney disease [1, 2]. Serum lipoprotein (a) $[\mathrm{Lp}(\mathrm{a})]$ has the homologous sequence with plasminogen. It can inhibit the fibrinolytic function on the surface of fibrin [3]. Plasminogen activator inhibitor-1 (PAI-1) is the primary inhibitor of plasminogen activators in plasma, and its high expression will also inhibit the dissolution of fibrin [4]. At present, LP (a) and PAI-1 are considered to associated with a variety of clinical diseases closely which are based on the injury of vascular endothelial cells $[5,6]$. However, the correlation of serum LP (a) and PAI-1 levels and clinical values of primary nephrotic syndrome (PNS) patients is not clearly. Therefore, this study for the first time analyzed not only the changes of $\mathrm{Lp}(\mathrm{a})$ and PAI-1 levels in PNS patients but also the correlation among plasma $\mathrm{Lp}(\mathrm{a})$, PAI-1 and albumin levels to further elucidate the possible mechanism of serum $\mathrm{Lp}(\mathrm{a})$ and PAI-1 in PNS patients.

\begin{abstract}
1 Materials and methods
1.1 Study subjects

The total of 60 patients with primary nephrotic syndrome for the study was recruited from the affiliated hospital of Qingdao university chose between April 2014 and July 2014. Their renal pathological types were identified according to the renal biopsy pathology diagnosis standard. Patients with diabetes mellitus, coronary heart disease, chronic hepatic disease, cancer, recent infection and so on which could affect lipid metabolism and fibrinolytic disorders were excluded. Secondary and hereditary nephrotic syndrome was also excluded. Fifty seven healthy people who had visited the affiliated hospital of Qingdao university to a routine check-up were selected randomly at the same period constituted the control group. The study protocol had been approved by the ethical committees of the hospital, and informed consent was obtained from all the recruiters. All laboratory measurements were performed after participants completed the study protocol.
\end{abstract}

This article is published under the terms of the Creative Commons Attribution License 4.0

Author(s) retain the copyright of this article. Publication rights with Alkhaer Publications.

Published at: http://www.ijsciences.com/pub/issue/2015-11/

DOI: 10.18483/ijSci.864; Online ISSN: 2305-3925; Print ISSN: 2410-4477 


\subsection{Methods}

\subsubsection{Collection of specimens}

Specimens of all the research objects were collected after an overnight fast for at 6 o'clock in the morning. First of all, the basic information of the research objects such as height, weight and so on were measured and recorded. Second, elbow venous blood about $3 \mathrm{ml}$ was extracted into the biochemical tubes, $37^{\circ} \mathrm{C}$ water bath for 15 minutes, and serum was promptly separated by a $20 \mathrm{~min}$ centrifugation at $2500 \mathrm{rpm}$ and maintained at $4^{\circ} \mathrm{C}$ before lipid, kidney parameters, and lipoproteins analysis. At the same time, $300 \mu 1$ upper serum of each specimen for PAI-1 measurement was kept at $-80{ }^{\circ} \mathrm{C}$ until analysis.

\subsubsection{Detection methods}

Serum levels of total protein, albumin (ALB), triglycerides (TG), total cholesterol (TC), low density lipoprotein cholesterol (LDL-c), urea nitrogen (BUN), creatinine (CREA) and $L p(a)$ were measured using commercial reagents by Hitachi 7600 automatic biochemical analyzer. Serum PAI-1 level was detected by a"sandwich"Enzyme-linked Immunosorbent Assay (ELISA). The human PAI-1 Elisa kits were bought from eBioscience. Each index of the detection had a strict standard of quality control, and tested by professional individuals who was trained and operated strictly according to the operation manuals.

\subsubsection{Statistical analysis}

Spss version 19.0 software was used to analyze the experimental data. One Sample Kolmogorov-Smirnov Test was used to evaluate the normality of distribution of the variables. The normal distribution data were expressed as mean \pm standard deviation, and skewed variables were expressed as median (interquartile range). The differences of variants of the two groups were analyzed by t-test when the data were consistent with a normal distribution. The differences of variants were analyzed by Wilcoxon rank sum test when the data were skewed distribution. The nonparametric Spearman rank coefficient test was used to analyze correlations between variables. Binary linear regression analysis was used to estimate risk factors of PNS patients. A two-tailed $\mathrm{P}$ value less than 0.05 was considered statistically significant.

\section{Results}

\subsection{Comparisons of general clinical information of group PNS}

The general clinical information in PNS and control group were shown in Table 1. There was no significant difference of gender, age, body mass index (BMI) of the two groups. Albumin levels were significantly lower in PNS than in the controls. Urea nitrogen level was significantly higher in PNS than $\mathrm{NC}$, but no significant change of creatinine level was noted between PNS and NC.

Table 1 Comparisons of general clinical information of group PNS

\begin{tabular}{|c|c|c|c|c|c|c|}
\hline Group & $\begin{array}{c}\mathrm{n} \\
\text { Male/Female }\end{array}$ & $\begin{array}{c}\text { Age } \\
\text { (years) }\end{array}$ & $\begin{array}{c}\text { BMI } \\
\left(\mathrm{kgm}^{-2}\right)\end{array}$ & $\begin{array}{l}\text { ALB } \\
\left(\mathrm{gL}^{-1}\right)\end{array}$ & $\begin{array}{c}\text { BUN } \\
\left(\mathrm{mmolL}^{-1}\right)\end{array}$ & $\begin{array}{c}\text { CREA } \\
\left(\mu \mathrm{molL}^{-1}\right)\end{array}$ \\
\hline PNS & $60(30 / 30)$ & $46.72 \pm 13.85$ & $25.21 \pm 3.36$ & $27.55 \pm 8.18^{*}$ & $5.72(5.05,7.57) *$ & $88.00(75.25,106.00)$ \\
\hline $\mathrm{NC}$ & $57(28 / 29)$ & $46.93 \pm 10.64$ & $24.36 \pm 3.85$ & $44.86 \pm 3.13$ & $4.71(4.30,5.27)$ & $85.00(75.00,92.00)$ \\
\hline$P$ & 0.924 & 0.926 & 0.217 & 0.00 & 0.00 & 0.15 \\
\hline
\end{tabular}

Compared with $\mathrm{NC}, * P<0.05$

2.2 Lp (a), PAI-1, lipids levels in the two groups

Lp(a), PAI-1 and lipids levels including triglycerides, total cholesterol and low density lipoprotein cholesterol were significantly higher in group PNS than in NC (Table 2).

Table 2 Lp (a), PAI-1, lipids levels in group PNS and NC

\begin{tabular}{cccccc}
\hline Group & $\mathrm{TG}\left(\mathrm{mmolL}^{-1}\right)$ & $\mathrm{TC}\left(\mathrm{mmolL}^{-1}\right)$ & $\mathrm{LDL}-\mathrm{c}\left(\mathrm{mmolL}^{-1}\right)$ & $\mathrm{LP}(\mathrm{a})\left(\mathrm{mgL}^{-1}\right)$ & PAI-1 $\left(\mathrm{ngmL}^{-1}\right)$ \\
\hline PNS & $1.88 \pm 0.88^{*}$ & $7.54 \pm 2.46^{*}$ & $4.28 \pm 1.90^{*}$ & $454.50(202.00,822.00)^{*}$ & $63.25(48.43,78.69)^{*}$ \\
NC & $0.91 \pm 0.38$ & $4.87 \pm 0.57$ & $2.72 \pm 0.34$ & $69.00(50.50,188.50)$ & $40.46(32.98,48.14)$ \\
$P$ & 0.00 & 0.00 & 0.00 & 0.00 & 0.00 \\
\hline
\end{tabular}

Compared with $\mathrm{NC}, * P<0.05$

2.3 Lp (a), PAI-1 and lipids related with albumin in PNS patients

Albumin was found negatively correlated with Lp(a), PAI-1, total cholesterol, triglyceride and LDL cholesterol levels in PNS patients (Table 3).

2.4 Correlation analysis of $\mathrm{Lp}$ (a) and PAI-1 in PNS group

Table 3 Correlation between plasma albumin and Lp(a), PAI-1, lipids in group PNS $\quad$ Lp(a)

\begin{tabular}{lcccccc}
\hline & TG & TC & LDL-c & Lp(a) & PAI-1 & was \\
\hline $\mathrm{r}_{\mathrm{s}}$ & -0.165 & -0.677 & -0.550 & -0.379 & -0.211 & posi \\
$P$ & 0.208 & 0.000 & 0.000 & 0.003 & 0.045 & tivel \\
& & & &
\end{tabular}

correlated with PAI-1 in PNS patients, and the Spearman rank coefficient was 0.381 ( $\mathrm{P}=0.003)$. No correlation was noted between Lp (a) and PAI-1 in normal control group.

2.5 The analysis of $\mathrm{Lp}(\mathrm{a})$ and PAI-1 with PNS patients 
Binary logistic regression analysis was performed to analyze weather Lp(a) and PAI-1 were risk factors in PNS patients. Consequently, we found that both Lp(a) and PAI-1 were two risk factors in PNS patients (Table 4).

Table 4 The analysis of Lp(a) and PAI-1 with PNS patients

\begin{tabular}{cccccccc}
\hline Variable & B & SE & Wald & $P$ & OR & \multicolumn{2}{c}{$95 \%$ CI } \\
\hline PAI-1 & 0.109 & 0.029 & 13.735 & 0.000 & 1.115 & 1.053 & 1.181 \\
Ln(a) & 0.010 & 0.002 & 16.823 & 0.000 & 1.010 & 1.005 & 1.015 \\
\hline
\end{tabular}

\section{Discussions}

Due to insidious onset of kidney disease when patients went to hospital the kidney disease has become seriously. Nephrotic syndrome as severe kidney disease affects the quality and safety of patients' life. The significant abnormalities of lipid metabolism and fibrinolytic system in patients with nephrotic syndrome are closely related to presence and severity of kidney disease[7-9].

Lp(a) was discovered a half-century ago by Kare Berg[10]. However, it still great challenges both basic researchers and clinicians. $\mathrm{Lp}(\mathrm{a})$ resembles a LDL-like particle that is linked to apolipoprotein(a) attached to apoB-100 by a disulfide bond[11, 12]. Elevated plasma levels of $\mathrm{Lp}(\mathrm{a})$ have been shown to be a independent risk factor for atherosclerosis [13, 14]. $L p(a)$ is difficult to grasp because of its unique structure, its considerable heterogeneity in both gene and isoform size, its wide range of plasma concentrations and its implication in ever more numerous cellular and biochemical mechanistic pathways[11]. In our study, we found that $\operatorname{Lp}(a)$ and lipids levels including triglycerides, total cholesterol and low density lipoprotein cholesterol were significantly higher in PNS than in the controls. Numerous studies have reported that the concentrations of total cholesterol, LDL cholesterol and triglyceride were elevated in NS patients [9, 15]. Hyperlipidemia and higher Lp(a) level may be caused by the increased hepatic protein synthesis driven by hypoalbuminemia and reduced clearance of $L p(a)$ in PNS patients[12]. Because albumin was found negatively correlated with $\mathrm{Lp}(\mathrm{a})$. Kidney disease causes lipid metabolic disorder and abnormal lipid metabolism speeds up the original kidney dysfunction in PNS patients. $\mathrm{Lp}(\mathrm{a})$ concentration remain extremely stable within an individual over their lifespan, and variation within the LPA gene on chromosome $6 \mathrm{q} 23$ contribute independently to the interindividual variability in plasma $\mathrm{Lp}(\mathrm{a})$ concentration[11, 16]. Therefore, further researches within the LPA gene of PNS patients are needed in the future.

The fibrinolytic system constitutes a critical response mechanism to vascular injury [17]. PAI-1 is an important inhibitor of the fibrinolytic system, so elevated levels could suppress fibrinolysis and result in an increased risk of thrombosis. PAI-1 is the primary inhibitor of plasminogen activators in plasma rapidly inactivating both tissue plasminogen activator (t-PA) and urokinase-type plasminogen activator (u-PA). So, PAI-1 inhibit t-PA converts the plasminogen into plasmin which plays a major role in fibrinolysis $[4,18]$. We found that PAI-1 levels are much more higer in PNS than NC group, which showed that patients with primary nephrotic syndrome has serious fibrinolytic system disorder. In our study, PAI-1 was found negatively correlated with albumin in PNS group. PAI-1 levels also may be caused by the increased hepatic protein synthesis driven by hypoalbuminemia. PAI- 1 is synthesized in the liver and by endothelial cells, and its synthesis is regulated by several physiologic mediators, including endotoxin, interleukin-1 and lipids [19], and PAI-1 gene also plays an important part in PAI-1 concentration [20]. These factors within PNS patients need to be further studies.

In conclusion, in our study the levels of $\mathrm{Lp}(\mathrm{a})$ and PAI-1 were increased in PNS group. Lp(a) and PAI-1 had already significantly changed before creatinine changes which response for renal function. They may be closely related to the presence and severity of kidney disease. $\mathrm{Lp}(\mathrm{a})$ was positively correlated with PAI-1 in PNS patients, we found that Lp(a) and PAI-1 were two risk factors in PNS patients. These findings provide a theoretical basis for the diagnosis and treatment of PNS.

\section{References:}

1) Pannier, B., et al., Postischemic vasodilation, endothelial activation, and cardiovascular remodeling in end-stage renal disease. Kidney Int, 2000. 57(3): p. 1091-9.

2) Malyszko, J., Mechanism of endothelial dysfunction in chronic kidney disease. Clinica Chimica Acta, 2010. 411(19-20): p. 1412-1420.

3) Gouni-Berthold, I. and H.K. Berthold, Lipoprotein(a): current perspectives. Curr Vasc Pharmacol, 2011. 9(6): p. 682-92.

4) Fortenberry, Y.M., Plasminogen activator inhibitor-1 inhibitors: a patent review (2006-present). Expert Opin Ther Pat, 2013. 23(7): p. 801-15.

5) Kamstrup, P.R., A. Tybjaerg-Hansen and B.G. Nordestgaard, Extreme lipoprotein(a) levels and improved cardiovascular risk prediction. J Am Coll Cardiol, 2013. 61(11): p. 1146-56.

6) Malgorzewicz, S., E. Skrzypczak-Jankun and J. Jankun, Plasminogen activator inhibitor-1 in kidney pathology (Review). Int J Mol Med, 2013. 31(3): p. 503-10.

7) Ghosh, A.K. and D.E. Vaughan, PAI-1 in tissue fibrosis. Journal of Cellular Physiology, 2012. 227(2): p. 493-507.

8) Eddy, A.A. and A.B. Fogo, Plasminogen activator inhibitor-1 in chronic kidney disease: evidence and mechanisms of action. J Am Soc Nephrol, 2006. 17(11): p. 2999-3012.

9) $\mathrm{Hu}, \mathrm{P}$., et al., Characteristics of lipid metabolism under different urinary protein excretion in children with primary nephrotic syndrome. Scand J Clin Lab Invest, 2009. 69(6): p. 680-6.

10) Berg, K., A New Serum Type System in Man-the LP System. Acta Pathol Microbiol Scand, 1963. 59: p. 369-82.

11) Dube, J.B., et al., Lipoprotein(a): more interesting than ever after 50 years. Curr Opin Lipidol, 2012. 23(2): p. 133-40.

12) Li, H.Q., et al., The level of native and oxidized 
lipoprotein(a) in children with nephrotic syndrome. Clin Biochem, 2012. 45(1-2): p. 101-5.

13) Enkhmaa, B., et al., Lipoprotein(a): genotype-phenotype relationship and impact on atherogenic risk. Metab Syndr Relat Disord, 2011. 9(6): p. 411-8.

14) Zlatohlavek, L., et al., Lipoprotein(a) and its position among other risk factors of atherosclerosis. Physiol Res, 2008. 57(5): p. 777-83.

15) Kronenberg, F., Dyslipidemia and nephrotic syndrome: recent advances. J Ren Nutr, 2005. 15(2): p. 195-203.

16) Kronenberg, F. and G. Utermann, Lipoprotein(a): resurrected by genetics. J Intern Med, 2013. 273(1): p. 6-30.

17) SOMERS, E.C., et al., Plasminogen Activator
Inhibitor-1 Is Associated with Impaired Endothelial Function in Women with Systemic Lupus Erythematosus. Annals of the New York Academy of Sciences, 2005. 1051(1): p. 271-280.

18) Hertig, A. and E. Rondeau, Plasminogen activator inhibitor type 1: the two faces of the same coin. Curr Opin Nephrol Hypertens, 2004. 13(1): p. 39-44.

19) Dimova, E.Y., A. Samoylenko and T. Kietzmann, Oxidative stress and hypoxia: implications for plasminogen activator inhibitor-1 expression. Antioxid Redox Signal, 2004. 6(4): p. 777-91.

20) Luo, Y., C. Wang and H. Tu, Impact of the $4 \mathrm{G} / 5 \mathrm{G}$ polymorphism in the plasminogen activator inhibitor-1 gene on primary nephrotic syndrome. Mol Med Rep, 2014. 9(3): p. 894-8. 